\title{
Transposition
}

Musique et Sciences Sociales

$7 \mid 2018$

Le prix de la musique

\section{Militer en chantant, sous l'œil de la police parisienne des années 1930 : une exploration du fonctionnement politique du chant}

Jonathan Thomas

\section{OpenEdition}

Journals

Édition électronique

URL : http://journals.openedition.org/transposition/1979

DOI : 10.4000/transposition. 1979

ISSN : 2110-6134

Éditeur

CRAL - Centre de recherche sur les arts et le langage

\section{Référence électronique}

Jonathan Thomas, « Militer en chantant, sous l'œil de la police parisienne des années 1930 : une exploration du fonctionnement politique du chant », Transposition [En ligne], 7 | 2018, mis en ligne le 15 septembre 2018, consulté le 14 novembre 2019. URL : http://journals.openedition.org/transposition/ 1979 ; DOI : 10.4000/transposition. 1979

Ce document a été généré automatiquement le 14 novembre 2019.

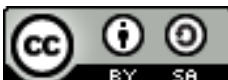

La revue Transposition est mise à disposition selon les termes de la Licence Creative Commons Attribution - Partage dans les Mêmes Conditions 4.0 International. 


\title{
Militer en chantant, sous l'œil de la police parisienne des années 1930 : une exploration du fonctionnement politique du chant
}

\author{
Jonathan Thomas
}

\section{Vers un imaginaire de la puissance politique du chant}

1 Quand Alain Corbin préface une vaste étude sur les usages politiques de la fête en France aux $\mathrm{xIX}^{\mathrm{e}}$ et $\mathrm{xx}^{\mathrm{e}}$ siècles, il commence par mettre en doute l'existence historique d'une fête exempte de toute instrumentalisation, «en quelque sorte, pure de toute politique $^{1} »$. Ainsi, plutôt que de proposer une histoire de la fête à laquelle il faudrait inclure des usages politiques à l'émergence insituable, mieux vaudrait puiser dans l'histoire la matière d'un "répertoire des modèles de fêtes politiques ${ }^{2}$ ». Cette démarche exemplaire en appelle une autre, contigüe, qui prendrait cette fois pour objet le chant et la dimension du sonore à laquelle il appartient, en contexte de pratique politique collective, c'est-à-dire de fête, de meeting ou de manifestation. En effet, quiconque s'est rendu à l'un de ces rassemblements y a sans doute entendu des chants ou des slogans exprimés sur quelques notes. Comme pour la fête, la première occurrence de l'association du chant à une pratique politique semble insituable, et la considération de toutes les autres inviterait à dégager le principe de cette association de toute historicité. Une histoire intégrale des usages du chant en contexte de pratique politique collective apparaît dès lors inenvisageable. Mais il reste possible de questionner la multitude des traces historiques de ces usages, et ainsi de comprendre comment le chant participe à l'histoire.

2 Un certain nombre d'ouvrages se sont déjà intéressés au chant comme «acteur de l'histoire " ", soit en relevant les effets remarquables et ponctuels de ses usages symboliques lors d'évènements politiques ${ }^{4}$, soit en s'attachant à un chant - la 
Marseillaise ou l'Internationale - pour écrire, en même temps que celle de ses chanteurs, l'histoire de ses usages contradictoires ${ }^{5}$ et questionner son fonctionnement symbolique ${ }^{6}$. Le champ d'une pragmatique du chant politisé est entrouvert par Michelle Perrot $^{7}$ ou Michel Vovelle ${ }^{8}$, qui relèvent le nombre d'exécutions publiques de différents chants politiques pour une période et un lieu. Philippe Darriulat propose une histoire des chansons politiques et sociales en France au XIX siècle, s'intéressant notamment à leurs contenus, leurs usages, leurs moyens de diffusion et leurs fonctions ${ }^{9}$. Enfin, le temps d'un chapitre, Daniel Morat interroge les affects provoqués par les chants politiques entonnés dans la rue et leurs effets ${ }^{10}$. Il est ainsi possible de proposer une démarche complémentaire à celles-ci, qui investira cette fois la question des intentions et des attentes d'effets relatives aux usages politiques du chant, celle de ses représentations chez ses acteurs et ses récepteurs, et qui cherchera à comprendre son fonctionnement en situation de pratique politique collective.

3 Au cœur de cette démarche se trouve une série de postulats formulés à partir d'un premier examen du corpus d'usages politiques du chant rassemblé pour cet article. Le premier de ces postulats est qu'il existe un nombre limité d'assemblages imaginaires basiques associant les engagements du chant dans des situations de pratique politique aux effets qu'en attendent ses chanteurs. Ces assemblages premiers prennent ici le nom de routines et sont la matrice pratique des usages politiques du chant. En conséquence, un deuxième postulat est que ces usages procèdent de conceptions du chant qui lui attribuent sa valeur politique en situation et circonscrivent ses capacités d'action, tout en les naturalisant. Ces conceptions sont partagées par les chanteurs et leurs récepteurs. Elles engagent les usages tout autant qu'elles sont engagées par eux, et sont une assurance relative que l'emploi d'un chant dans une situation donnée aura l'effet recherché. Un troisième postulat est que routines et conceptions partagées du chant procèdent d'un imaginaire de la puissance politique du chant, par lequel sont organisés et reçus ses usages politiques selon leurs situations. Cet imaginaire, par lequel se constituent les usages, est aussi constitué par leur expérience acquise, transmise puis sédimentée. En ce sens, cet imaginaire évolue, et doit être situé dans le temps et l'espace. Cette démarche devra donc dégager ces routines à partir d'une situation temporelle et géographique particulière, pour aborder un imaginaire de la puissance politique du chant.

4 Pour ce faire, des fêtes, des réunions et des manifestations politiques tenues à Paris, pour la plupart entre 1934 et 1938, seront considérées ici. Ce choix se justifie autant par l'intensité particulière que prend l'exploitation du son par des pratiques politiques collectives, que par des conditions historiques favorables à la multiplication des faits de chant, en effet nombreux et décisifs. Partis politiques et militants s'approprient alors largement des technologies sonores encore récentes. Le disque est adopté par la SFIO et l'Action française en 1929, suivies par la CGT (1930) et le Parti communiste (1932) pour diffuser discours et chants, partout où se trouve un phonographe ${ }^{11}$. Il s'ajoute au hautparleur, dont le premier usage politique semble dater, en France, de $1924^{12}$, et dont la banalisation au cours des années 1930 met l'accent sur la valeur politique de l'impérialisme sonore. Par ailleurs, ces nouvelles pratiques se tiennent alors que la France connaît un climat politique agité, dont la tension culmine autour des émeutes insurrectionnelles du 6 février 1934. Ces manifestations mortelles de ligues d'extrêmedroite et d'associations d'anciens combattants provoquent la chute du gouvernement en place et sont alors perçues comme une tentative manquée de coup d'État. Elles provoquent en retour l'union antifasciste des partis socialiste et communiste. Celle-ci 
fait de Paris le théatre quotidien de manifestations ${ }^{13}$, où le chant se fait entendre dans des situations d'opposition militante parfois violentes. Les meetings et les fêtes se déroulent également en nombre et rassemblent souvent plusieurs milliers de personnes. Le chant y est encore présent, soit qu'il est entonné par l'assistance, soit qu'il est confié à des chorales populaires, des artistes lyriques ou des disques. Tout concourt ainsi à faire des années 1930 une décennie particulièrement sonore, voire bruyante. Elle l'est d'autant plus en comparaison des années de l'Occupation allemande. L'usage politique du chant y est alors surtout le fait du gouvernement de Vichy, qui cantonne les manifestations chantées adverses dans la clandestinité de leurs chanteurs, lesquels résistent en chantant bas, brièvement et par petits groupes, des airs alors jugés, dans leurs bouches, séditieux ${ }^{14}$.

5 Le corpus ainsi situé nous est rendu accessible par les comptes rendus - établis ex post et les brefs rapports - établis in situ - produits par la Police municipale parisienne et la Direction des renseignements généraux et des jeux ${ }^{15}$, au cours de leurs missions de surveillance politique et de maintien de l'ordre. Il s'agit de sources précieuses à plus d'un titre, malgré leur justesse et leur niveau de précision parfois médiocre. Elles offrent déjà les programmes musicaux, les descriptions des ambiances sonores, des déroulés des fêtes et des manifestations, ainsi que des faits de chants surveillés. Mais elles sont surtout les traces de l'écoute policière qui participe à les produire. Cette écoute est un moyen à la fois unique, indirect et explicite de figuration des pouvoirs politiques du chant et de la puissance d'agir qu'il donne à ses chanteurs. Par elle, les policiers tirent des sons le moyen d'apprécier l'état de la situation surveillée, puis, à partir des effets sociaux - constatés ou redoutés - des sons écoutés, le moyen d'anticiper l'évolution de cette situation. Comptes rendus et rapports de surveillance rendent ainsi possible de relever les usages politiques du chant, d'en dégager les types et de formuler les routines à leur origine. Ils suggèrent d'abord ce que pourrait être la partie policière d'un imaginaire de la puissance politique du chant mais, puisqu'ils décrivent les pratiques des militants chantant, ils font de même pour la partie militante de cet imaginaire. Ces deux parties qui peuvent être conçues comme contraire l'une à l'autre, puisqu'elles sont relatives à deux forces mises en oppositions, procèdent pourtant d'un même imaginaire du chant comme moyen d'action dans une situation de pratique politique. Le chant représente alors pour les militants et les policiers une puissance d'agir que les premiers doivent libérer, et les seconds contenir.

Cette source documentaire nourrira une réflexion exploratoire qui cherchera donc à se figurer d'abord la partie policière de l'imaginaire de la puissance politique du chant pour ensuite aborder sa partie militante, notamment à travers les pratiques et mises en œuvre du chant par les chefs politiques et leurs affiliés. Elle commencera par se figurer pourquoi et comment la police doit surveiller les rassemblements politiques, puis elle cherchera à comprendre les caractères des pratiques d'écoute policière dans différents contextes de pratique politique, afin de pouvoir cerner les types d'usages politiques du chant, leur valeur d'agir et les intentions qui les engagent. Elle relèvera ensuite différents faits de son instrumentalisation par des chefs politiques en posture d'orateur, pour finir par se concentrer sur l'un de ces faits - l'association de la Marseillaise à l'Internationale par le Parti communiste -, et interroger cette occurrence particulière $d u$ fonctionnement symbolique du chant en politique. Celle-ci et les analyses déployées tout au long de ce texte nous permettront enfin de dégager les routines à l'origine des usages politiques du chant. 


\section{Les tâches policières et leurs terrains}

7 Tenter d'approcher, à travers le regard policier, ce que peut être un imaginaire de la puissance politique du chant nécessite de se figurer comment la police évalue sa valeur subversive, c'est-à-dire l'intensité de ses effets et leurs incidences sur les missions policières. Cette approche implique en premier lieu de mesurer les enjeux de la surveillance politique qu'assure la police parisienne. Celle-ci est placée sous l'autorité du Préfet de Police de Paris, qui doit prévenir et disperser les «attroupements et réunions tumultueuses ", et préserver la "liberté et la sûreté de la voie publique ${ }^{16}$ ». Ces missions tirent leur importance de la concentration des institutions du pouvoir étatique dans une ville à la longue histoire insurrectionnelle. L'historien de la police Jean-Marc Berlière note ainsi que la police parisienne est, plus qu'une autre, cadenassée par le pouvoir central, qui la conçoit comme le moyen de contrôle d'une population depuis longtemps réputée séditieuse :

Cette méfiance du pouvoir central à l'égard du peuple de Paris, nous la retrouvons sous la III République, exacerbée par le souvenir de la Commune. Toutes les révolutions et tous les changements de régime ont eu lieu à Paris, les gouvernants de la III République sont bien placés pour le savoir et c'est ce constat qui justifie pour une grande part la pérennité sous un régime républicain d'une institution qu'il n'aurait, semble-t-il, pas dû tolérer ${ }^{17}$.

La surveillance des rassemblements politiques parisiens n'a donc pas pour seul but la préservation d'un ordre public que viendraient troubler des affrontements verbaux ou physiques. À travers la récolte de renseignements sur le nombre et la ferveur des militants, la popularité ou l'impopularité des chefs politiques, le contenu de leurs discours et de leurs appels à l'action, c'est donc bien la perspective, même lointaine, d'un renversement du pouvoir en place qui préoccupe la police. Et chaque terrain de surveillance, chaque type de rassemblement possède ses caractères propres, qui dictent à la police ses modalités d'attention et d'action. La fête ou le meeting, relativement circonscrits à un endroit bien délimité, peuvent se montrer avant tout comme des indicateurs de la probabilité d'un tel événement. En revanche, c'est dans la rue que s'effectue le passage à l'acte des révolutions ou des manifestations violentes que peuvent préparer les réunions sédentaires. En effet, «il n'existe de régime stable que celui qui tient la rue à Paris. [...] C'est là que la République joue son existence, c'est le rôle du Préfet de Police et de la Police municipale de la protéger; pour cela, le contrôle quotidien de la rue est la première de leurs missions ${ }^{18}$ ». Et cette mission justifie notamment une surveillance rapportée presque minute par minute par tout un réseau d'agents de la police parisienne - et consignée par écrit.

Pour comprendre la mission de surveillance de la police et aborder au mieux l'écoute policière, il convient donc de différencier fête, meeting et manifestation politique sans toutefois s'appuyer sur une typologie trop rigide, et alors trompeuse, de leurs productions formelles. En effet, rien que les manifestations de rue de l'été 1936 empruntent déjà à la fête ses représentations fantaisistes, ses parodies et ses rituels ${ }^{19}$. Plus d'une fête et d'un meeting politique pourraient par ailleurs confondre leurs déroulés : la fête est toujours l'occasion d'une prise de parole politique; le meeting accueille souvent une partie musicale, chantée ou non. En revanche, manifestation, fête et meeting peuvent être distingués par la catégorie de leur cadre spatial de 
déroulement. Pour informer cette distinction, nous nous appuierons sur les catégories de lieu et d'espace, telles que les a définies Michel de Certeau ${ }^{20}$.

Le meeting, d'abord, est une pratique sédentaire qui se tient dans un lieu. Celui-ci est pour de Certeau l'endroit où "les éléments considérés sont les uns à côté des autres, chacun situé en un endroit "propre" et distinct qu'il définit ${ }^{21}$ ». Ici, c'est l'injonction d'efficacité d'une pratique politique dans sa phase de transmission des signes politiques et d'accumulation d'énergie qui fixe les modalités d'occupation d'un endroit. Elle y décide des mouvements licites et illicites, établit une hiérarchie parmi les occupants de la salle, désigne les chefs et ceux, ne pouvant bouger sans leur assentiment, qui doivent les suivre. Ainsi, les orateurs sont à la tribune et font face à l'assistance. Ils rendent asymétriques les échanges tenus dans la salle, en monopolisant une parole rendue écrasante par l'emploi réservé d'un micro et de haut-parleurs. Leur public doit se tenir dans des endroits où il ne peut apparaître que comme un ensemble homogène d'adhérents à un projet politique. Ses seules possibilités d'expression - chanter, crier le font d'autant plus apparaître comme force brute, ou comme masse. La pratique politique du meeting ne peut tirer de bénéfices de la constitution de son endroit en lieu que par sa stabilisation durant le temps de la transmission des messages partisans. Les éventuels contradicteurs de ces messages, envoyés par d'autres partis, pourraient contrarier cette organisation en tentant de briser l'unité d'adhésion militante et l'ordre qu'elle instaure entre la masse et l'orateur. Mais ils se montrent aussi comme des occasions d'affirmer encore la force de la stabilité de l'occupation de l'endroit en lieu, par la réponse qu'apporte l'orateur et qui doit réduire la contradiction à l'ineptie ou au silence.

Caractéristique du meeting, la stabilité d'occupation du lieu est absente de l'espace, que de Certeau conçoit comme "un croisement de mobiles, [...] l'effet produit par les opérations qui l'orientent, le circonstancient, le temporalisent et l'amènent à fonctionner en unité polyvalente de programmes conflictuels ou de proximités contractuelles $^{22} \%$. En contexte de manifestation, la rue est un espace, et une pratique politique collective peut espérer y trouver des bénéfices d'une autre nature que dans un lieu $^{23}$. Mais elle est un espace inachevé. Elle fait l'objet d'une concurrence de production d'espaces entre les manifestants - qui essaient d'en faire celui de leur hégémonie politique -, et la police - qui tente de contrer cet essai pour ramener la rue à sa configuration de voie publique.

Quant à la fête, elle semble devoir relever d'une succession d'organisations en lieu, puis en espace, selon que l'assistance écoute, fixée, un orateur ou qu'elle danse lors du bal suivant la délivrance des indispensables messages politiques. Restant confinée dans l'espace d'une salle, elle ne représente toutefois pas un enjeu sécuritaire équivalent à celui de la manifestation.

La rue et la salle de meeting ou de fête apparaissent donc comme trois terrains déterminant différentes formes de mobilisations politiques. La salle ${ }^{24}$ est le lieu d'emprise de l'orateur, qui peut y moduler l'état affectuel de son assistance, grosse de plusieurs centaines ou milliers de personnes. Isolée du monde extérieur, elle semble être avant tout, lors du meeting, le lieu de la production et de la concentration d'une énergie politique latente et, lors de la fête, celui de la socialisation utopique d'un projet politique, qui peut alors imprimer son empreinte sur des échanges sociaux par ailleurs étrangers à la pure activité militante. La rue, en monde à s'approprier, est l'espace d'expression d'un état individuel et collectif médiatisé et intensément travaillé par le 
politique; une expression qui peut être comprise comme la libération de l'énergie politique auparavant accumulée dans les rassemblements enclos dans des lieux.

Les sources policières de cette étude indiquent que les surveillances exercées s'adaptent à ces différences d'endroits. Elles sont, dans l'enceinte des salles, un moyen de renseignement sur l'état des discours et des tactiques politiques, d'évaluation de la ferveur militante et de la popularité des orateurs. Dans la rue, leur mission de renseignement doit aider au contrôle des foules et au maintien de l'ordre. Leurs premiers moyens sensibles sont la vue - les comptes rendus et les rapports détaillent la présence des drapeaux, emblèmes, portraits, etc. Mais ces sources indiquent aussi que l'écoute est un moyen incontournable de la surveillance policière, et qu'elle est adaptée aux conditions spatiales des surveillances exercées. Elle se signale d'abord dans les sources par la présence très fréquente d'informations relatives aux sons, et plus particulièrement au chant - moment des chants, titres, chanteurs, réception par le public militant, les manifestants ou les passants. Ensuite, l'articulation de ces informations à d'autres faits déjà déroulés ou redoutés montrent que les usages politiques du chant sont particulièrement surveillés, et donc craints, par les policiers, en tant qu'ils pourraient participer à compromettre leurs missions. Les sources mobilisées ici nous offrent donc en premier lieu un accès à la façon dont la police écoute les usages politiques du chant, puis un accès à la partie policière d'un imaginaire situé de la puissance politique du chant. Il est donc maintenant temps de les explorer et de commencer leur analyse, pour commencer d'établir et de remonter la chaine qui nous mènera aux éléments de cet imaginaire.

\section{Les traces d'une écoute policière}

15 Tout d'abord, comment et à quelles occasions le chant apparaitt-il dans les archives policières? Il faut commencer par noter qu'il est mentionné pour lui-même, hors de toute articulation avec de possibles désordres, aux côtés d'autres informations comme la situation géographique des personnes surveillées, leur nombre, leur appartenance politique, leur éventuelle constitution en groupes, les symboles visuels qu'ils convoquent - drapeaux, emblèmes, insignes, gestes - leur état d'excitation, leur engagement dans des actes de vandalisme ou dans des affrontements verbaux ou physiques. Dans les rapports relatifs à quatre manifestations d'ampleurs diverses tenues entre le 14 juillet 1935 et le 18 mars 1937, le chant entonné est une simple information contextuelle : "La tête du cortège est arrivée à la hauteur de la rue des Boulets; les manifestants chantent des hymnes révolutionnaires ${ }^{25}$.»; " Le $7^{e}$ groupe arrive place de la Bastille. Les participants chantent l'Internationale ${ }^{26}$.»; "Actuellement le calme est revenu. Les participants chantent "L'Internationale" et le "Ça ira" ${ }^{27}$ »; « Un autre groupe de cinq cents manifestants, en marche dans la direction de l'Opéra, occupe le tiers de la chaussée et chante "La Carmagnole", "l'Internationale" et réclame sur l'air des lampions: "Des canons pour l'Espagne." ${ }^{28}$ ». Sans qu'il soit toujours annonciateur de troubles, le chant est donc déjà considéré comme un élément de première importance pour appréhender l'état d'un espace public alors en ballotage d'appropriation. Il semble gagner une valeur subversive supplémentaire et engager un surcroît d'attention policière quand il est rapporté aux côtés des " cris ", c'est-à-dire des slogans hurlés par les manifestants ${ }^{29}$, et ce d'autant plus que leurs absences sont elles aussi relevées - «ni chants, ni cris $^{30} »$. 
la brutalité de l'un semble devoir briser la patiente construction de l'autre, et non amplifier sa force. Bien qu'ils existent tous deux dans la dimension sonore des manifestations, le chant et le cri sont ici situés aux antipodes de l'esthétique et de l'imaginaire social de la voix. Alors que le cri est une manifestation individuelle ou collective saturée, quasi-monotone, précipitée, assourdissante et finalement violente, le chant est le fruit d'un travail réflexif et nuancé d'apprentissage et de raffinement de la maîtrise d'une voix modulée sur plusieurs hauteurs et différentes dynamiques. Quand le cri ne nécessite des crieurs aucune compétence particulière autre que celle de la copie, la mise en œuvre du chant requiert des chanteurs de ne pas être faux, ainsi que la connaissance collective de sa mélodie et de son texte. Et si le chant peut aussi reproduire l'impulsion du cri pour gagner un moyen de mobilisation politique efficace, en s'appropriant son effet performatif propre ${ }^{31}$, il peut être pensé comme un anti-cri, ou $\mathrm{du}$ moins comme un outil politique sonore plus élaboré, plus complexe à mettre en œuvre et, le cas échéant, mobilisable comme un signe de légitimité et un moyen d'élévation culturelle, notamment dans le lieu du meeting ou de la fête ${ }^{32}$. Mais en contexte de manifestation - dans la rue qui n'est certes ni l'opéra, ni le cabaret, et se prête difficilement à la délectation esthétique du chant - la police ne considère pas, à de rares exceptions près ${ }^{33}$, les qualités plastiques de son interprétation. Articulé au cri, le chant politisé n'est, pour elle, plus du tout un objet d'art, et aucun des rapports consultés n'implique ses qualités esthétiques dans la survenance ou la crainte de la survenance d'incidents. L'association du chant avec le cri, ce produit de la volonté d'un agir politique immédiat et inconditionnel, est le signe que la perception de ses qualités esthétiques est recouverte par la qualité performative de son usage politique. La perception policière du chant dégage donc son esthétique de l'explication de ses effets, où ne se tient plus que le simple fait de sa survenance. Toutefois, il est alors courant que l'harmonie actancielle des chanteurs soit reconnue et instrumentalisée comme une petite société utopique. Le groupe des manifestants chantants pouvait être ainsi perçu comme l'émergence menaçante d'un germe social coordonné, dont le programme d'action s'entendrait dans les paroles du chant ou dans l'histoire de ses exécutions publiques.

Ainsi, pour comprendre pourquoi les archives de la police mentionnent tant de fois le chant et pour ordonner ces mentions, nous pourrions introduire l'idée d'une gradation policière de sa capacité de nuisance. Si la simple mention du chant et son association aux cris ne traduisent pas encore de danger imminent, elles prennent un sens différent si on considère que le chant peut rassembler plus de chanteurs, amplifier les affects des manifestants et leur mobilisation ${ }^{34}$, pour enfin provoquer des incidents. Ces derniers peuvent être attendus, mais ne pas survenir: "Quelques cris et chants retentissent mais sans provoquer d'échos ${ }^{35}$ »; "Les participants font entendre des chants révolutionnaires notamment la "Carmagnole" et l'“'Internationale". Pas d'incident ${ }^{36}$. ». Mais ils peuvent aussi se réaliser. Lors de la manifestation du 22 juin 1936, à la Gare Saint-Lazare, où se font face militants du Front populaire et ceux du Front National une confédération des ligues françaises consécutive aux événements du 6 février 1934 le chant participe peut-être à faire d'une opposition symbolique un bref pugilat :

L'agitation atteint son maximum vers $19 \mathrm{~h} 45$, heure à laquelle les manifestants étaient divisés en deux camps d'importance sensiblement égale. Les partisans du «Front Populaire » [...] chantaient «L'Internationale », « La Jeune Garde » et criaient "Vive le Front Populaire», «La Rocque au poteau». Les partisans du 
« Front National » criaient «La France aux Français » et «Vive La Rocque ». Des coups ont été échangés entre manifestants. Cinq d'entre eux ont été contusionnés, mais sans gravité, ainsi que deux gardiens de la paix ${ }^{37}$. Michel Chion. Il propose, à partir des travaux de Pierre Schaeffer, trois modes d'écoute : causale, sémantique, réduite ${ }^{40}$. L'écoute réduite, désignant une écoute concentrée sur le son pour lui-même, n'est pas ici un outil d'investigation adéquat, tant elle ne transparait pas dans les archives consultées. Ce n'est pas le cas de l'écoute causale, « la plus commune, [qui] consiste à écouter un son dans le but d'obtenir une information sur sa cause (ou sa source) ${ }^{41}$ ", laquelle semble apte à soutenir un effort d'évaluation de l'état d'une situation et de ses possibilités d'évolution. Il en va de même pour l'écoute sémantique, qui s'attache à déchiffrer ce qu'elle perçoit d'un langage. Elle pourrait même précéder une écoute causale, puisque les services de police semblent prendre en considération le contenu textuel d'un chant pour confirmer sa politisation et évaluer son potentiel de nuisance. Ainsi, alors que la police est attentive aux éventuelles reconstitutions de l'Action française et de ses associations peu après leur dissolution en février 1936, le Gardien de la Paix André Rage surveille une permanence des Étudiants d'Action française et rapporte ceci :

Au cours de ma surveillance, je n'ai rien remarqué d'inhabituel [...]; cependant, vers $17 \mathrm{~h} \mathrm{30}$, un certain nombre [de jeunes gens] [...], se sont mis à chanter un air entrainant dont je n'ai pu comprendre les paroles [...]. Ce chant a duré environ 15 minutes; je ne suis pas intervenu et comme tout se passait à l'intérieur de l'immeuble, je n'ai pas cru devoir en rendre compte ${ }^{42}$. 
21 Si Rage rapporte finalement qu'il n'a pas rendu compte de ce fait de chant, c'est bien parce qu'en l'absence d'un contenu sémantique perceptible, il ne l'a pas compris. Le défaut de son écoute sémantique aurait pu le faire passer à côté d'une violation de la loi, comme le rapporte par ailleurs le commissaire d'arrondissement André Torlet ${ }^{43}$, plus attentif que l'agent à la valeur comme signe de ce chant incompris. Alors que les traces d'une existence clandestine de l'Action française sont recherchées par la police en février et mars 1936 pour s'assurer de sa dissolution effective, le chant est bien perçu comme l'emblème d'un groupe qu'il consolide encore comme acte, au même titre que les insignes portées par les militants, ou les enseignes accrochées aux façades et aux fenêtres des immeubles parisiens, qui se rapportent aux associations d'Action française ${ }^{44}$.

Dans cet exemple, les paroles du chant doivent le rattacher sans aucun doute possible à un groupe politique pour achever de constituer son usage politique. Ainsi, l'écoute sémantique articulée au répertoire des chants politiques donne d'abord au chant une valeur de signe identitaire, par ailleurs constitué et reconnu hors du cercle policier. Mais ce signe sonore n'est jamais complètement stabilisé, et n'est donc pas toujours univoque. Danielle Tartakowsky s'appuie sur lui pour tenter de démêler l'écheveau des appartenances politiques revendiquées en chantant par les militants et les émeutiers des événements du 6 février 1934. En rappelant les associations de la Marseillaise aux anciens combattants, de l'Internationale aux communistes de l'Association républicaine des anciens combattants (ARAC), de la Madelon aux Croix-de-feu, mais en rappelant aussi que cette dernière, ainsi que le Chant du départ et la Marseillaise, peuvent être entonnés par des " organisations de droite très diverses, voire de simples passants ${ }^{45}$ ", elle donne autant une idée des forces en présence que, à travers leur vraisemblable cacophonie, de la confusion sonore caractéristique de cette soirée d'émeutes. La corrélation que permet l'écoute sémantique entre la signifiance identitaire du chant, la reconnaissance des forces politiques en présence et la signification de son usage politique peut donc être trompeuse, et renforcer d'autant plus cette idée que le chant est une matière sonore contagieuse.

Trompeuse, l'écoute sémantique l'est encore en amenant les policiers à déchiffrer les paroles des chants politiques comme des programmes d'action, et à entendre ainsi L'Internationale, La Jeune Garde ou la Marseillaise comme les annonciateurs d'une grande violence. Si cette hypothèse paraît extravagante, elle s'accorde pourtant bien d'une part avec la reconnaissance de la capacité de ces chants à provoquer la sédition et la révolution, et de l'autre avec la persistance de cette reconnaissance, malgré la proportion extrêmement faible des révolutions et des émeutes déclenchées par rapport à leurs exécutions très fréquentes. Leurs effets peuvent même être inverses de ceux attendus par la police. Par exemple, la Marseillaise - ce chant qui appelle à prendre les armes et à tuer - chantée par l'Union des anciens combattants (UNC) au soir du 6 février 1934, n'excite pas plus des émeutiers dont les violences, en face de l'Assemblée nationale, semblent insurrectionnelles, mais au contraire les apaise ${ }^{46}$. L'écoute sémantique montre ainsi qu'elle ne peut suffire à la police pour acquérir une expérience utile des situations de manifestation, mais qu'en reconnaissant toujours un pouvoir insurrectionnel au chant, elle participe bien à la partie policière de l'imaginaire de sa puissance politique.

Les émeutes et les manifestations du 6 février mettent enfin particulièrement en valeur la nécessité, pour tous leurs acteurs, de la mise en œuvre conjointe d'une écoute 
causale et d'une écoute sémantique, pour leur permettre d'assembler des informations sur un état du présent, sur ses possibilités d'évolution, et leur faire percevoir des possibilités d'agir selon leurs propres intérêts. L'une et l'autre s'assemblent alors pour former une "écoute praticienne ${ }^{47}$ ", forgée par l'expérience et orientée vers la captation du panel des informations utiles à une pratique donnée. Un des points sensibles de l'enquête consécutive aux émeutes mortelles du 6 février est de savoir si oui ou non, des sonneries au clairon et des sommations verbales ont bien été exécutées - et si elles ont été audibles -, avant que les forces de l'ordre tirent sur les émeutiers ${ }^{48}$. La conventionalité qui constitue sonneries au clairon et sommations en avertissements est à saisir ici dans le cadre d'une telle écoute, dans ce cas partagée entre policiers et manifestants. Elle repose alors sur un répertoire spécifique d'assignations communes de sons à des causes et à ce qu'ils annoncent. Néanmoins, si l'avertissement s'établit comme l'élément d'un langage partagé et doit ainsi procéder d'une écoute praticienne commune, les tâches policières procèdent d'une écoute praticienne étrangère à celle des manifestants, tout en étant portée sur les mêmes signes d'usages et en reconnaissant leur valeur politique. Il convient maintenant d'investir cette écoute policière praticienne en passant par le biais de l'écoute causale, pour relever les usages politiques du chant en manifestation, quand ils participent à des oppositions militantes parfois violentes et font du chant une arme symbolique.

\section{Contrôler ceux qui chantent contre}

Si l'écoute sémantique est utile pour reconnaître, à partir des paroles d'un chant, une appartenance politique, l'écoute causale construit quant à elle le répertoire de ses observations à partir d'un constat basique : si un agent entend un chant où il reconnait plusieurs voix mêlées et ajustées, c'est qu'un groupe le chante. Et, alors qu'il est pourtant essentiel, et donc habituel, à la manifestation de " "faire groupe", constituer une "totalité", "prendre corps" ${ }^{49}$ ", le groupe chantant semble devoir faire l'objet d'un surcroit de soupçons. Peut-être est-ce parce qu'un groupe est perçu comme plus vaste quand il use d'instruments de musique et $\mathrm{crie}^{50}$ ou, peut-on ajouter, qu'il chante, montrant ainsi les limites de la véridicité d'une écoute causale. Peut-être est-ce parce que « le bruit promet le renfort que l'on espère, et [que] c'est un augure favorable pour les actes qui se préparent ${ }^{51} "$, alors que le chant existe comme le bruit dans la dimension du sonore. Toujours en suivant l'auteur de Masse et puissance, un autre décalage serait possible. Si, comme il l'écrit, «le feu [...] est visible de loin et attire les autres $^{52}$ ", il pourrait en être de même pour le chant, audible de loin, attirant «les autres » qui le connaissent, partageant son contenu sémantique et une certaine partie de son histoire symbolique. Il pourrait alors fonctionner comme ce que Murray Schafer nomme un son centripète ${ }^{53}$, attirant à lui des individus dispersés qui renforceront un groupe toujours plus important. Le groupe chantant pourrait ainsi fonctionner comme ce que Canetti nomme un cristal de masse, l'un et l'autre partageant la capacité de "déclencher la formation de masses " et, jusqu'à un certain point, leur caractère délimité et persistant ${ }^{54}$. Cristal de masse et groupe chantant ont ainsi surtout en commun «la netteté, l'isolement et la constance [qui les font se détacher] de façon inquiétante sur les phénomènes d'agitation de la masse elle-même ${ }^{55}$ ». Le groupe chantant, éventuel cristal de masse, possède des propriétés imaginaires qui provoquent dans tous les cas leur surveillance. Celle-ci n'aboutit cependant pas toujours à sa dispersion, dont les circonstances ne sont pas toujours renseignées dans les rapports de 
police, et dont les motifs sont donc peu clairs et semblent aléatoires. Ainsi, lors d'une manifestation de protestation, le 16 février 1936, contre l'agression de Léon Blum par des sympathisants de l'Action française, les groupes chantants repérés semblent n'être pas dispersés par la police. Peut-être est-ce parce qu'ils semblent se déliter d'euxmêmes ${ }^{56}$ ou qu'ils occupent un espace déjà désigné et aménagé dans ce but par les autorités publiques? Un groupe de 3000 personnes chantant l'Internationale devant un kiosque à musique de la Place de la Nation n'est en tous cas que signalé, sans que sa dispersion soit renseignée ${ }^{57}$, alors que ce même jour, un groupe de 200 personnes chantant la Marseillaise est dispersé sans incident ${ }^{58}$. En revanche, quand un militant du Parti social français (PSF) est poursuivi par des membres du service d'ordre de la manifestation de Front populaire du 14 juillet 1937 et qu'il se réfugie dans un café, le groupe qui stationne devant et chante l'Internationale n'est pas dispersé ${ }^{59}$, alors qu'il pourrait être impliqué dans cet incident et faire pression sur le militant de droite. Un dernier cas confirme toutefois que le groupe chantant est particulièrement visé par la police et que la décision de sa dispersion est suffisamment fréquente pour être attendue et contrée par les manifestants :

Le 13 juillet [1935] à 22 heures, le Gardien SIMON Pierre a remarqué qu'un groupe d'une cinquantaine de personnes parcourait en chantant "l'Internationale" les rues François-Miron et Tiron [...]. Avertit, l'inspecteur principal JAPPEL se rendit sur les lieux avec le car Secours-Police et rejoignit le groupe [...]. À la vue du car les manifestants se dispersèrent rapidement et non seulement les gardiens n'eurent pas à employer la force mais ils ne purent procéder à aucune arrestation ${ }^{60}$.

La constitution de groupes chantants se montre donc comme le préalable, semble-t-il reconnu comme tel par la police, à un panel de pratiques que permet ou catalyse le chant, qui sont fondamentales des pratiques politiques collectives, et que la police peut être amenée à empêcher. D'après les archives consultées, la plus saillante d'entre elles est la revendication d'une identité politique, entrainant la démarcation des groupes et des espaces. Son corollaire immédiat est l'occupation symbolique d'un territoire dont l'aire n'est pas délimitée par les corps des chanteurs mais par l'étendue sonore du chant lui-même. Dans cette perspective, le comportement d'un groupe d'étudiants de droite, lors de la manifestation de Front populaire du 16 février 1936, étonne et désigne peut-être une des modalités de l'usage territorial du chant. Un rapport mentionne en effet que ce groupe a " tenté de se mêler à la foule en chantant "La Marseillaise" ${ }^{61}$ ", à la suite d'une opposition verbale avec un autre groupe d'étudiants aux opinions politiques adverses. Effectuée sans discrétion, donc, cette tentative de se mêler à la foule ne semble pas destinée à dissoudre le groupe pour préserver ses membres d'éventuelles violences physiques, mais bien à étendre leur territoire symbolique par l'instauration d'îlots de chants qui en sont autant de bornes sonores et rayonnantes, et qui pourraient aussi leur permettre de se gagner la foule.

Cet usage tactique raffiné du chant se montre comme une modalité du combat symbolique qu'il permet d'engager. En manifestation, avant d'en venir éventuellement aux mains, les militants de camps politiques opposés s'affrontent d'abord à coup de cris, d'insultes, mais aussi de chants. Le 22 juin 1936, à la gare Saint-Lazare, la Marseillaise et l'Internationale sont chantées par deux groupes entre lesquels se déroulent des « incidents ${ }^{62}$ ». Le 12 juillet 1936, un petit groupe de jeunes militants de gauche traversent la Gare Saint-Lazare en chantant la Jeune garde. Alors qu'ils prennent un taxi, quatre jeunes militants de droite entament, semble-t-il pour les provoquer, une Marseillaise $^{63}$. Le 4 octobre 1936, alors que des militants du PSF se préparent à 
manifester contre la tenue d'un meeting du PCF au Parc des Princes, instruction leur est donnée d'éviter de trop faire monter la tension, et de la cantonner au terrain symbolique et incontestable du chant: « Pas de provocations inutiles, mais on répondra à "L'Internationale" par "La Marseillaise". Recommandation est faite de crier "Vive l'Armée !" ${ }^{64}$ ". Ces bagarres de chants sont d'ailleurs autant de jalons d'une histoire des assignations symboliques et des appropriations variables des chants entonnés par chaque camp. Elles témoignent de leur instabilité quand, le 16 février 1936, un groupe de jeunes militants de droite répondent - provoquant l'intervention immédiate de la Police municipale - à l'Internationale de quelques militants du Front populaire par une Marseillaise pourtant chantée par les manifestants de gauche tout au long de la journée ${ }^{65}$. Mais le chant n'est pas qu'un moyen d'opposition, qui débouche sur des violences et motive ainsi les interventions policières. En plus d'être valorisé par l'imaginaire policier comme un facteur de troubles et anticipé pour cette raison, il l'est aussi comme un indicateur de la qualité des relations des militants avec leurs partis, de leur engagement et de leur adhésion. L'écoute praticienne des manifestations doit alors être accompagnée de celle qui évalue la situation des meetings, ou des manifestations stabilisées qui transforment leurs espaces - comme de grandes places parisiennes - en lieux, pour permettre la captation par tous de la parole de l'orateur. Les archives de la police se montrent alors, encore, autant comme des rapports de surveillance que comme des traces de ce qui fait outil politique dans le chant, pour ses chanteurs.

\section{Surveiller ceux qui chantent pour}

28 Un des principaux usages politiques du chant est ainsi d'exprimer et d'amplifier l'adhésion et l'engagement militant. Dans les manifestations de gauche des 14 juillet 1935 et 24 juin 1936, la présence et les discours de chefs politiques sont salués par des Internationale qui sont autant de marques de reconnaissance du charisme des orateurs et d'approbation de leurs discours. L'usage fait du chant pour acclamer une figure politique pourrait être conçu ici comme mixte sur le plan spatial, car il survient autant dans l'espace de la rue que dans le lieu du meeting ou de la fête. Dans ces contextes spatiaux, l'acclamation par le chant tient même, par sa fréquence, autant du moteur que de la trame du rassemblement politique. Considérant de nombreux comptes rendus de fêtes ou de meeting politiques, notamment de gauche, le chant - ou la musique semble devoir toujours occuper le temps de son établissement, quand l'assistance prend sa part de l'organisation de la salle en lieu, et se prépare à recevoir la parole des orateurs. Le chant ou son air sont ainsi exécutés par un orchestre ${ }^{66}$ ou diffusés par un phonographe relié à des haut-parleurs ${ }^{67}$. Le chant peut être repris au refrain par le public, avant l'accueil du premier intervenant ${ }^{68}$. Il est par ailleurs fréquent que le public militant de gauche entonne une Internationale à la mesure de son estime pour celui-ci, en ouverture puis en clôture de son discours, et répète ce rituel à chaque nouvel orateur $^{69}$. Le meeting est ainsi mis en scène par la musique, mais c'est bien le chant montant de l'assistance qui soutient le rythme et l'intensité de son déroulement, et finit par le constituer en succès ou en échec.

29 Et le meeting s'apparente peut-être vraiment à une fête quand chant et musique ne sont plus le fait du public, mais d'orchestres, de chœurs, de chanteuses et de chanteurs lyriques qui peuvent interpréter aussi bien des chants politiques que des airs d'opéra. Si la question de la signification politique des pièces et de la provenance sociale de leurs 
interprètes se pose, elle est trop vaste et laissera ici sa place à celle portant sur la justification de leur choix. Car l'écoute de la police n'est plus ici causale et prédictive, mais principalement sémantique, relevant les titres des chants, des airs, des pièces et les noms de leurs compositeurs ${ }^{70}$. Elle considérerait donc les détails du répertoire joué, même s'il lui paraît, à tort, étranger à tout usage politique possible ${ }^{71}$, comme des renseignements à part entière, pourquoi pas utiles aux lecteurs de ces rapports pour se figurer une signification politique aux musiques jouées.

Ainsi, la police ne manque pas de relever qu'au cours d'une fête de charité organisée par les «Dames royalistes", la chanteuse Josefina Attard, interprète entre autres "l'hymne franquiste ${ }^{72}$ ». Le compte rendu de cette fête, qui fait apparaître une seconde fois cette information à droite du corps du texte et la constitue ainsi comme un des renseignements à retenir de cette soirée ${ }^{73}$, indique également que la chanteuse tenait dans ses bras un bouquet évoquant l'Espagne de Franco, « cravaté aux couleurs sang et or [et que] cet hymne a été écouté par les assistants debout et a été longuement applaudi ${ }^{74}$ ». Un an plus tôt, la police anticipe que «le chanteur nègre Paul Robson se fera entendre dans son répertoire ${ }^{75}$ » lors d'une soirée qu'organisera le Comité national allemand pour l'aide à l'Espagne, le 11 février 1938. La présence du chanteur semble confirmée par le compte rendu de la soirée, bien que celui-ci soit entretemps devenu pour la police le " baryton nègre Robinson ${ }^{76}$ ", alors qu'il est en fait l'acteur et chanteur américain Paul Robeson, à la voix de basse et aux multiples engagements politiques de gauche. La police fait ainsi émerger la question des usages politiques de la musique et du chant non plus par des manifestants, mais par des organisations et des chefs politiques, à travers le répertoire et la personnalité des interprètes qu'ils convoquent ${ }^{77}$ - autant d'indices d'une histoire des politiques culturelles partisanes.

31 Mais la police relève également, de la part des orateurs ou des chefs, plusieurs autres faits notables d'instrumentalisation du chant, à fins d'agir sur l'état affectuel d'une assistance à qui il est demandé de chanter. Il s'agit alors toujours de conclure le meeting, par ce qui ressemble à un rituel où le chant occupe une place que les exemples suivants permettront de définir. Le 13 février 1936, à la fin d'une soirée artistique (environ 1500 personnes) du Mouvement social français Croix de Feu, précurseur du PSF, le délégué général à la propagande Charles Vallin définit ce qu'est le Mouvement, « recommande à ses auditeurs d'avoir toujours une confiance aveugle dans le colonel de la Rocque et les autres chefs et [...] termine en leur demandant de crier deux fois : "Vive le Rocque ! Vive la France !" puis de chanter la Marseillaise ${ }^{78}$ ». Ici, le chant - associé au cri non plus par la police, mais par les acteurs politiques qu'elle surveille - est utilisé pour sa capacité à consolider une adhésion. La même année, le 28 octobre, devant un millier de personnes, un délégué à la propagande du PSF, fondé en juillet, termine son intervention par la lecture d'un tract appelant à une contre-manifestation des militants d'extrême-gauche, qui veulent ainsi répondre au rassemblement de ces militants qu'ils considèrent comme fascistes. Il poursuit :

Le 13 juillet [1935] à 22 heures, le Gardien SIMON Pierre a remarqué qu'un groupe d'une cinquantaine de personnes parcourait en chantant "l'Internationale" les rues François-Miron et Tiron [...]. Averti, l'inspecteur principal JAPPEL se rendit sur les lieux avec le car Secours-Police et rejoignit le groupe [...]. À la vue du car les manifestants se dispersèrent rapidement et non seulement les gardiens n'eurent pas à employer la force mais ils ne purent procéder à aucune arrestation ${ }^{79}$.

Là, le chant est demandé par l'orateur alors qu'est exposée la mise en cause de son identité politique et de celle de l'assistance, qu'est attendue et suscitée en retour leur 
confirmation, et que l'extérieur de la salle de meeting pourrait être l'espace d'affrontement de volontés d'altération physique des identités politiques de chaque camp. Le chant fonctionne encore comme un moyen de consolidation d'une adhésion, cette fois non dans le but de la constitution, mais de la résistance du groupe à ce qui tente de le dissoudre. Ce surcroit d'engagement de chacun - pris dans la «mystique PSF » - dans la survie du groupe confine à un effacement de l'individu dans sa consécration à une figure de l'Autre qui le subsume. Le groupe politique prend une dimension à la fois divine et terrestre. S'il existe ici une "mystique PSF ", c'est bien parce que la mobilisation politique tend à se confondre avec l'engagement religieux, en même temps que la politique emprunte en quelque sorte sa liturgie à la religion ${ }^{80}$. La proximité, voire la confusion, des rassemblements PSF avec les pratiques rituelles religieuses, autres signes d'un projet de fabrication sociétale, est d'ailleurs, selon Danielle Tartakowsky, un trait caractéristique des fêtes de droite en général, consécutif des liens unissant la droite et l'église ${ }^{81}$. Ainsi, le 30 mars 1940, pour clore l'assemblée d'une soixantaine de personnes de la section PSF du Bel-Air-Picpus, son chef M. Thomas « fait une dernière fois appel à "la foi P.S.F." et [invite] les assistants à entonner "La Marseillaise" ${ }^{22} »$.

Alors qu'un orateur fait appel à la foi de son assistance, et lui demande de chanter pour, dans un même mouvement, l'invoquer et y confirmer l'inscription de tous les membres chantants du groupe - les silencieux se trahissant - il faut reconnaître la part du chant dans ce rituel de clôture où le politique semble trouver une part non négligeable de sa puissance d'action. Ces trois exemples de rituels chantés interviennent en effet alors que le groupe politique, rassemblé et irrigué par la parole et les affects suscités par le ou les orateurs, est sur le point de se séparer, et que sa situation actuelle laisse un doute sur son avenir. C'est alors un moment d'angoisse, car après la fête ou le meeting, en dehors de la salle, quand viendra le moment où les militants pourront répandre et faire grandir les germes d'action politique qu'ils viennent de recevoir, leur masse sera atomisée. Se constituera-t-elle à nouveau, lors d'une autre réunion, plus large et puissante, pour rapprocher un peu plus le projet politique qui la rassemble de sa réalisation? Réapparaitra-t-elle affaiblie? Ou ne réapparaitra-t-elle jamais? Par ailleurs, quel sera le degré d'engagement militant de chacun une fois qu'il se sera extrait du monde essentiellement politique qui configure ce qui advient dans la salle de meeting ou de fête? Le chant semble alors intervenir comme un remède à cette angoisse, par un pacte de chacun avec tous scellé par le partage de la sémantique, alors rendue unique, d'un même texte résonnant dans une mélodie qui en désigne les pleins et les déliés émotionnels et qui doit faire ressentir à chacun, au même moment, les mêmes affects. C'est cette manière de faire groupe par communauté émotionnelle, ou par communion dans la vérité que l'émotion donne à son objet - ici, n'importe quelle cause politique - que semblent chercher les chefs politiques, qui peuvent ainsi trouver un biais supplémentaire à leurs talents oratoires pour agir sur leur assistance.

Mais l'exemple suivant semble encore révélateur d'une autre dimension des effets du chant sur un groupe. Le 31 juillet 1932, Jacques Doriot termine de s'exprimer devant 5000 militants communistes réunis au Stade de l'Unité, à Saint-Denis. Ceux-ci n'ont pas reçu l'autorisation du gouvernement de manifester au sortir du meeting. Conscient de la frustration de son assistance, Doriot lui dit :

Mais nous en ferons d'autres, ajoute-t-il, ici ou ailleurs, en plein air. Sans renoncer à notre droit à la rue, je vous demande de sortir dans le calme. Nous manifesterons quand nous estimerons que l'heure en sera venue. Vous sortirez donc par petits 
groupes, tout cortège étant interdit, et vous suivrez les deux itinéraires indiqués dans le journal l'Humanité, d'un côté le Barrage, de l'autre la route du fort. Je vous demande également de ne pas répondre à aucune des provocations qui ne manqueront pas de vous être faites à la sortie. Je vous autorise cependant à terminer cette manifestation au chant de l'Internationale ${ }^{83}$.

Doriot tente de faire passer la manifestation interdite pour une manifestation reconnue comme inappropriée dans ce contexte, et donc de faire d'une interdiction dictée depuis l'extérieur une volonté des militants et de leur chef de ne pas manifester. Il essaie de prévenir ainsi la possible colère de son assistance afin d'éviter tout incident, même en cas de provocations lancées à des individus qui seront forcés à n'être plus des militants, mais de simples passants. L'autorisation finale qu'il donne de chanter l'Internationale fait alors penser que le chant fonctionne comme le médiateur que chacun pourra convoquer pour se décharger de sa colère et de ses affects, mais encore que ceux-ci auraient pu être plus puissants et dangereux si le chant n'avait pas été autorisé. Et ils auraient pu l'être non seulement parce que le chant n'aurait pu assurer sa tâche de décharge, mais aussi parce qu'il semble désiré pour sa capacité de représentation symbolique d'une société en devenir, et pour sa capacité d'autojustification, qu'il permet en tant que preuve, travail et emblème du but utopique qui motive l'êtremilitant. Or, cette sorte particulière d'appétence de la masse des militants pour le chant politique permet de penser un peu plus le chant comme un moyen mis en œuvre par les chefs politiques pour réguler, selon les termes fluctuants de leurs tactiques, les affects et l'orientation de l'action des militants. Il est alors concevable comme un puissant moyen pour rassembler, en premier lieu, une masse militante, mais aussi, pourquoi pas, tout un peuple. C'est ainsi qu'un dernier cas doit être évoqué ici pour continuer d'informer l'imaginaire de la puissance politique du chant, depuis sa partie militante. Il montre la manœuvre simultanée de plusieurs masses militantes par une opération symbolique portée sur le menu de leurs chants emblématiques, alors que se scelle l'union capable d'amener trois anciens adversaires politiques au pouvoir.

\section{La Marseillaise avec l'Internationale : un cas de braconnage et d'unification symbolique par le chant}

Le 14 juillet 1935 doit avoir lieu une grande manifestation du rassemblement antifasciste des partis socialiste et communiste qui, augmenté pour la première fois du Parti radical, débouchera sur la création d'un Comité national pour le rassemblement populaire. Ce comité, qui lie classes moyenne et ouvrière, est le précurseur du Front populaire. À cette occasion, le PCF veut que ses militants adjoignent la Marseillaise à leur traditionnelle Internationale. Le 5 juillet 1935, dans la salle des fêtes de la mairie de Bagnolet, un orateur du parti communiste en informe les futurs manifestants :

[Beckler] fait enfin diverses recommandations en ce qui concerne ce rassemblement. "Il faut, dit-il, que la manifestation se déroule dans le calme et la dignité. "Nous chanterons "la Marseillaise" avec la pensée qu'à l'origine cet hymne était révolutionnaire et le drapeau tricolore, emblème de victoire sur la royauté, figurera à côté du drapeau rouge. Lorsqu'on entonnera "la Marseillaise" il ne faudra pas tenter de l'étouffer en chantant "l'Internationale", mais la chanter de tout cœur, en alternant avec l'“Internationale" ${ }^{4}$.

Beckler prépare en fait son assistance à faire quelque chose qu'elle semble ne pas avoir à cœur de faire ou qui, du moins, ne lui semble pas naturel. Internationale et Marseillaise 
ont en effet, depuis la fin du xix siècle, une histoire commune de proximités et d'éloignements striée de frictions ${ }^{85}$, et il n'y a pas que les militants de la droite qui pourraient être scandalisés par un rapprochement opéré entre les symboles de la Nation française - drapeau et chant - et ceux du communisme international ${ }^{86}$. Au début des années 1930, avant la manœuvre initiée par Maurice Thorez pour donner au Parti communiste la légitimité nationale nécessaire à son accès démocratique au pouvoir ${ }^{87}$, l'extrême-gauche n'aimait pas la Marseillaise, qu'elle "considérait encore comme l'expression barbare d'un chauvinisme dépassé ${ }^{88} »$. C'est alors surtout une symbolique de la pureté nationale, et donc du nationalisme, constamment convoquée par certains des chanteurs de la Marseillaise au lendemain de la Première Guerre mondiale, qui l'oppose à l'Internationale. Celle-ci est en effet le symbole d'une lutte de libération qui doit transcender les nations pour s'adresser à l'humanité entière. Cette assignation négative du symbole de la nation tournée vers elle-même - et donc de celui d'une prochaine guerre - à la Marseillaise fait contraste et valorise en retour l'Internationale comme un hymne de la fraternité planétaire. Ainsi, pour saluer le départ de Paul Robeson, son assistance entonne l'Internationale, chant "commencé en allemand, continué en français et terminé en espagnol ${ }^{89}$ ». Pourtant, leurs histoires montrent aussi que les symboliques respectives de l'Internationale et de la Marseillaise sont suffisamment contigües pour que le texte de la première ait été conçu sur la musique de la seconde ${ }^{90}$, que Jean Jaurès, dans un texte de 1903, considère l'Internationale comme la « suite prolétarienne de la Marseillaise ${ }^{91}$ » et que cette dernière soit d'abord chantée, en février 1917, par ceux qui adopteront, deux mois plus tard, l'Internationale comme hymne pour continuer la révolution en Russie.

Si l'adoption par des militants de gauche et d'extrême gauche de la Marseillaise ne va alors pas de soi, elle reste envisageable et sa difficulté ne rebute pas les chefs du Parti communiste, dont la pugnacité pose la question de leurs attentes de bénéfices politiques. Si ceux-ci sont clairs, les mécanismes qui les produisent et leurs modalités de réalisation méritent d'être détaillés. Il faut déjà revenir sur la possibilité même, pour le Parti communiste, de proposer la Marseillaise à ses militants sans perdre sa crédibilité auprès d'eux. Celle-ci est en effet, comme le manifestent les événements du 6 février 1934, la propriété des organisations politiques de droite et des associations d'anciens combattants $^{92}$, qui veillent au respect de l'idéal républicain et français pour lequel ils se sont battus. Mais cette appropriation particulière de l'hymne national est, comme d'autres, instable et passagère. L'histoire mouvementée des appropriations et des assignations symboliques de la Marseillaise est déjà permise par ce que Buch reconnaît comme « un fonctionnement typique [du chant politique] : celui d'un signe lancé dans le champ politique susceptible d'être approprié et adapté par chacun à son propre discours ${ }^{93}$ ». Il est possible d'avancer un peu plus dans l'intelligibilité de cette opération d'appropriation en considérant qu'un chant partage avec une langue la capacité de se faire le signe de symboles, que la Marseillaise est, en tant qu'hymne national depuis 1879, un signe répandu et connu par tous sur tout le territoire de la Nation ${ }^{94}$, qu'il est certes l'objet d'interprétations diverses, mais que sa légitimité à symboliser la société française apparaît d'autant plus comme une convention bien établie que certains la remettent en cause. La polysémie de la Marseillaise, ou pour le dire autrement la diversité de ses assignations symboliques, en fait alors un matériau de choix pour mettre en œuvre une stratégie politique de rassemblement. Nous pouvons alors suivre Pierre Bourdieu, pour qui «la religion et la politique tirent leurs meilleurs effets idéologiques des possibilités qu'enferme la polysémie inhérente à l'ubiquité sociale de 
la langue légitime ${ }^{95} »$. La légitimité historique, nationale et populaire de la Marseillaise la désignent pour travailler classes moyenne et ouvrière selon le sens que chacune lui reconnait, effacer la distance qui les sépare, les ramener sous le giron d'un même chant et de la nation qu'il représente, pour faire socle à un nouveau gouvernement populaire. Mais elle permet encore autre chose. La concurrence d'appropriation qu'instaure le Parti communiste entre les forces de gauche et les « fascistes » peut aussi être comprise comme l'exercice de la possibilité de réinvestissement symbolique qu'offre le chant, pour diminuer ou parasiter l'imaginaire convoqué par le discours politique de l'ennemi fasciste. Le Secrétaire général du PCF Maurice Thorez déclare ainsi le 22 janvier 1936, lors du $8^{e}$ Congrès du parti :

Sur le plan idéologique, nous avons fourbi les nouvelles armes de la classe ouvrière tout en reprenant à l'ennemi ce dont il nous avait soustrait; chantant notre Internationale, nous avons repris la Marseillaise ; brandissant notre drapeau rouge, nous avons repris le drapeau tricolore. Nous avons repris les strophes sur la liberté et appliqué au fascisme, ennemi du peuple de France, les paroles de Rouget de L'Isle : "Ils viennent jusque dans nos bras égorger nos fils, nos compagnes" ${ }^{96}$.

Le Parti communiste n'a par ailleurs pas à faire trop de contorsions pour intégrer la Marseillaise à son patrimoine symbolique et l'associer à la classe ouvrière. Il lui suffit en effet de la reconnaître, cette fois-ci en France, avant tout comme le symbole du renversement de la monarchie par la République, et donc comme celui de la libération populaire. Par ailleurs, le contexte politique et international de cette opération pourrait la rendre fructueuse. Alors que la menace des régimes nazi et fasciste résonne dans l'action des ligues françaises d'extrême-droite, et que se succèdent en moins de dix-huit mois quatre gouvernements tous incapables d'offrir les conditions d'une vie abordable aux Français, redonner à la Marseillaise sa charge symbolique révolutionnaire, populaire, humaniste et la faire chanter dans la rue revient à rejouer symboliquement la Révolution, selon son imaginaire de libération de la misère et de l'oppression - fut-elle dorénavant capitaliste - et ainsi d'accession à des conditions matérielles utiles au bonheur. Le Peuple est ainsi de nouveau en armes de ce chant qu'il s'est réapproprié, en circonscrivant ses possibilités d'assignation symbolique par sa mise en miroir avec l'Internationale.

Et la Marseillaise est alors aussi et surtout mobilisée comme un symbole national, et non plus nationaliste, dans un contexte de manifestation où il participe et donne sa qualité à " cette opération de légitimation que seul le défilé dans son actualité peut effectuer ${ }^{97}$ ». Son appropriation est ainsi avant tout celle d'une légitimité nationale, c'est-à-dire d'une légitimité à faire nation. Ce qui deviendra le Front populaire doit en effet, pour gagner le pouvoir, être représentatif de la nation française en même temps que de son peuple, aux contradictions sociales résolues. Le nouvel usage politique de la Marseillaise semble apte à répondre à cette nécessité, qui désigne la priorité de l'établissement d'une hégémonie politique symbolique avant celle d'une hégémonie institutionnelle. Et l'appropriation se fait, tout comme l'usage fonctionne. De la grande manifestation antifasciste du 14 juillet 1935 à celle victorieuse du 14 juillet 1936, la Marseillaise devient en effet «l'hymne retrouvé du Front populaire ${ }^{98}$ ». Ce dernier aurait-il été possible et victorieux si seul le drapeau tricolore avait été mobilisé pour symboliser la centralité de la nation française dans le projet politique de l'union des gauches? Il y aurait peutêtre eu un malentendu si, le 14 juillet 1935, place de la Bastille, seule l'Internationale avait été entendue parmi les 500000 personnes présentes. Cela aurait signifié qu'elles souhaitaient porter leur projet politique d'élection sur l'humanité entière, pour 
remporter la «lutte finale », et qu'en visant l'humanité et la victoire dans la dernière des luttes, elles ne limitaient pas leur désir de libération aux frontières d'une communauté particulière, soumise à ses propres relations de domination, réclamant ses propres modalités de libération. Ce faisant, elles auraient cantonné la portée de leur projet politique à une action purement symbolique, sans qu'il puisse être concrétisé par son exercice sur un objet à sa portée, car circonscrit et différencié. De fait, si l'Internationale a permis à ceux qui ont aussi chanté la Marseillaise de délimiter le sens de cette dernière et de ne pas la donner à comprendre comme celle que chantaient les militants de droite et d'extrême-droite, la Marseillaise leur a permis d'attacher le projet de libération porté par l'Internationale à une communauté imaginée particulière, nationale, présentant ses propres problèmes à résoudre. Et de légitimer ainsi la saisie symbolique de ces problèmes et l'orientation politique de leur résolution dans la perspective du message de l'Internationale. Ce faisant, les masses militantes et chantantes participant à former le Front populaire ont ouvert une perspective d'application localisée et circonstanciée à leur projet politique, rapprochant ainsi le rêve de la réalité. Et grâce au chant, mobilisé par des partis de masses pour elles, elles ajoutent à la contextualisation nécessaire à la mise en œuvre effective d'un projet politique, la force de leur rassemblement en une communauté imaginée, qui fond dans son identité nationale son identité politique, et peut manifester à ses ennemis le symbole triomphant, hégémonique, de la force que lui donne la cohésion d'une identité commune. La communauté chantante peut ainsi gagner et construire le monde comme s'il était le sien, c'est-à-dire le seul qui vaille.

\section{Les routines d'un agir politique par le chant}

41 Il faut, pour finir cette analyse des usages politiques du chant, dégager quelques routines de sa manipulation en situation de pratique politique collective, qui sont autant de signes de l'imaginaire de sa puissance politique. La première d'entre elles est fondamentale. Elle est déduite de la présence du chant dans les rapports et les comptes rendus policiers et de sa justification: s'il y figure, c'est qu'il participe à produire la dangerosité politique des situations surveillées et qu'il possède donc une valeur pour un agir politique. La première de ces routines est ainsi qu'entonner un chant dans une situation de pratique politique collective produira un effet qui participera à l'évolution de cette situation, selon la volonté et les intérêts du ou des chanteurs.

Dans le cas où le groupe chantant chercherait à fonctionner comme un cristal de masse, le fait que le chant ne provoque pas toujours les effets politiques souhaités par ses chanteurs met d'autant plus l'accent sur la constitution imaginaire des pouvoirs qui lui sont prêtés. L'imaginaire de sa puissance politique forme ainsi les modalités d'intervention du chant sur le monde non pas par le calcul et l'évaluation froide de ses probabilités de succès, mais dans le moule d'un fantasme de maitrise où la volonté d'un acteur peut se réaliser avec fluidité, comme par magie. Cet imaginaire est donc aussi celui d'un acte de communication, qui chiffre par le chant une intention que d'autres de ses connaisseurs pourraient déchiffrer sans perte de sens, et qui leur indiquerait quoi faire. Par exemple, Marc Ferro rapporte qu'en 1917 :

La lutte pour la paix était devenue l'objectif numéro un de la propagande bolchevik.

Dans la Pravda, Lénine avait recommandé aux soldats russes qui étaient dans les tranchées de chanter l'Internationale: les soldats allemands ou autrichiens d'en face, pour peu qu'il y ait des ouvriers parmi eux, ne manqueraient pas d'en être 
émus, et ainsi, de fraternisation en fraternisation, les soldats lèveraient la crosse en l'air'99. symbolique d'un chant serait à même de générer et d'orienter ses effets politiques pour les rendre utiles à une pratique, selon les exigences stratégiques du moment, comme dans le cas de l'association de la Marseillaise à l'Internationale. L'exemple de Ferro nous permet par ailleurs de développer cette routine. Il y est attendu que le chant fonctionne non pas pour causer la révolte de tous les soldats contre la hiérarchie oppressive qui les envoie mourir au combat, mais bien comme un signe d'appartenance à la même classe sociale, transcendant les appartenances nationales. Ce n'est donc pas l'entièreté du programme politique de l'Internationale que mobilise Lénine, mais une partie de sa signifiance possible. La musique peut suffire à la porter, si sa référentialité avec l'univers symbolique des paroles est suffisamment constituée et répandue. Elle rend même son signifiant plus facilement perceptible dans ce contexte, où des paroles chantées vraisemblablement en russe à des soldats germanophones avaient déjà à couvrir et à franchir les sons et les espaces de la guerre. Il peut dès lors s'instaurer un jeu d'interactions symboliques entre une musique à la référentialité déjà construite et un texte différent de son texte d'origine, jusqu'à - et souvent pour - la contradiction. Il existe ainsi de nombreuses Marseillaises, dont une royaliste, la Marseillaise des Blancs. La polysémie essentielle du chant politique conditionne alors une troisième routine de sa manipulation politique, reprenant l'assertion de Buch citée plus haut: un chant peut être approprié et mobilisé par n'importe qui en sera capable, quel que soit son ancrage politique et les buts qu'il poursuit, dans la perspective stratégique d'une appropriation symbolique plus large et dommageable pour les propriétaires actuels du chant. Cette routine permet la mise en scène musicale de l'inscription politique des chanteurs militants dans une situation particulière, dépassant les contradictions d'assignation identitaire d'un chant particulier pour en tirer un bénéfice politique unique. Ce faisant, elle s'ajoute aux précédentes pour nous suggérer que les usages politiques du chant sont moins gouvernés par son hypothétique essence symbolique que par les exigences d'une situation, qui ne cessent de pousser les chanteurs à travailler le matériau symbolique chanté. Chaque chant se montre ainsi comme un pur instrument, dont l'équipement symbolique peut être adapté pour répondre à des usages certes variant en situation, mais qui peuvent être définis par une typologie large. Chacun de ces usages est issu de la confrontation qu'opèrent les militants, dans une volonté d'agir immédiate, entre les caractères de leur situation présente et les routines de manipulation du chant.

Ainsi, trois types d'usages ont été relevés au cours de cette étude : ceux qui visent à rassembler un groupe, le rendre reconnaissable par autrui tout en lui conférant un territoire face à un groupe adverse ; ceux qui visent à exprimer et à faire communion avec autrui, notamment l'orateur ou le chef politique ; ceux qui visent à appuyer et à exalter un processus d'engagement symbolique et physique. Ces usages peuvent être considérés comme les organes d'une fonction générale de territorialisation spatiale, politique et identitaire, assurant au cristal de masse chantant l'endroit de son existence et de son développement. Le chant agirait ainsi comme un moyen d'occupation symbolique qui se confond, par sa dimension sonore, avec un moyen d'occupation physique. Et cette conception territoriale du chant l'amène à être considéré dans la perspective de ce qu'est, d'une façon générale, l'occupation d'un territoire : un espace que l'on s'approprie, dont l'appropriation est disputée jusqu'à la mort, mais qui ne 
cesse d'être changeante, sur lequel se construisent, perdurent puis disparaissent des identités différentes et adverses. C'est bien cette lutte pour la possession de l'espace qui fait relever à la police les faits de chant en contexte de manifestation. Et s'il faut finir ici par la tentative d'informer ce que pourraient être les parties policières et militantes d'un imaginaire de la puissance politique du chant pour la police, il faut déjà rappeler l'hypothèse que pour la police, le groupe chantant apparaisse comme l'émergence d'un ordre social adverse dans le chaos de la manifestation, une organisation sociale dont le chant montre la coordination et l'union nécessaire à la conquête du pouvoir. Pour les militants, le chant serait, par son exécution, le moyen de se constituer en un groupe à l'identité revendiquée, voire en un cristal de masse, apte à réaliser l'objet final de sa mobilisation. Il serait alors connexe d'un imaginaire de la révolte et de la conquête, et ainsi de la libération. Mais pour les chefs politiques, il serait un moyen supplémentaire à la parole pour réguler l'état affectuel de la masse militante, pas seulement par l'exaltation que le sentiment d'adhésion et d'union lui procure, mais par la stratégie gouvernant le moment de sa demande ou de son autorisation. Il serait ainsi suffisamment puissant pour, comme le laisse entendre Jacques Doriot quand il retient son audience de former un cortège mais l'«autorise cependant à terminer cette manifestation au chant de l'Internationale ${ }^{100}$ ", tenir une masse par la gestion de sa frustration et mesurer l'emprise du chef sur ses militants, c'est-à-dire le degré de leur aliénation. Son imaginaire serait alors connexe de celui du contrôle, et sa dimension esthétique résonne avec celle des totalitarismes des années 1930. Le chant politisé apparaît ainsi comme le plus beau moyen d'expression d'une volonté de façonner le meilleur des mondes, tout en étant agi pour poser des frontières et délimiter insidieusement la liberté de tous, à commencer par celle de ses chanteurs.

\section{BIBLIOGRAPHIE}

\section{Bibliographie}

ALTEN Michèle, « Un siècle d'enseignement musical à l'école primaire », Vingtième Siècle, revue d'histoire, $\mathrm{n}^{\circ}$ 55, 1997, pp. 3-15.

BECKER Jean-Jacques \& BERSTEIN Serge, Histoire de l'anti-communisme en France, Paris, Orban, 1987. BERLIÈRE Jean-Marc, Le préfet Lépine : vers la naissance de la police moderne, Paris, Éditions Denoël, 1993.

BERSTEIN Serge, Le 6 février 1934, Paris, Gallimard/Juillard, 1975.

BOURDIEU Pierre, Langage et pouvoir symbolique, Paris, Le Seuil, 2001.

BUCH Esteban, La Neuvième de Beethoven : une histoire politique, Paris, Gallimard, 1999.

BUCH Esteban, « Les hymnes », DUCLERT Vincent \& PROCHASSON Christophe (dir.), Dictionnaire critique de la République, Paris, Flammarion, 2007, pp. 896-901.

CANETTI Elias, Masse et puissance, Paris, Gallimard, 1966. 
CERTEAU Michel DE, L'invention du quotidien. Tome 1: Arts de faire, Paris, Gallimard, 2015.

CHION Michel, « The three listening modes », STERNE Jonathan (dir.), Sound Studies Reader, Oxon, Routledge, 2012, pp. 48-53.

CORBIN Alain, « Préface », CORBIN Alain, GÉRÔME Noëlle \& TARTAKOWSKY Danielle (dir.), Les usages politiques des fêtes aux 19-20 siècles, Paris, Publications de la Sorbonne, 1994, pp. 7-11.

DARRIULAT Philippe, La Muse du peuple : chansons politiques et sociales en France 1815-1871, Rennes, Presses Universitaires de Rennes, 2010.

FERRO Marc, L'Internationale : histoire d'un chant de Pottier et Degeyter, Paris, Agnès Viénot, 1996.

MARIN Louis, De la représentation, Paris, Le Seuil, 1994.

MERCIER Arnaud, «Efficacité du performatif dans les rituels politiques », Hermès, La Revue, vol. 3, n 43, 2005, p. 31-37, en ligne.

MORAT Daniel, « Cheer, Songs, and Marching Sounds : Acoustic Mobilization and Collective Affects at the Beginning of World War I ", MORAT Daniel (Dir.), Sounds of Modern History : auditory cultures in $19^{\text {th }}$ - and $20^{\text {th }}$ - century Europe, New-York/Oxford, Berghahn Books, 2017 [2014], pp. 177-200.

ORY Pascal, « De "Ciné-Liberté" à La Marseillaise : espoirs et limites d'un cinéma libéré (1936-1938) », BOUVIER Jean (dir.), La France en mouvement 1934-1938, Seyssel, Champ-Vallon, 1986, pp. 276-297.

PERROT Michelle, Les ouvriers en grève : France 1871-1890 (2 Vol.), Paris, Mouton \& Co, 1974, 900 p. QUÉNIART Jean, Le chant, acteur de l'histoire, Rennes, Presses universitaires de Rennes, 1999, 359 p. SCHAEFFER Pierre, Traité des objets musicaux : essai interdisciplines, Paris, Le Seuil, 1966. SCHAFER Murray, Le paysage sonore : le monde comme musique, Paris, Wildproject, 2010. SIAUD Florent, «Liturgies de la rue », Tracés. Revue de Sciences humaines, vol. 5, 2004, en ligne. TARTAKOWSKY Danielle, « Stratégies de la rue 1934-1936 », BOUVIER Jean (dir.), La France en mouvement 1934-1938, Seyssel, Champ-Vallon, 1986, pp. 31-60.

TARTAKOWSKY Danielle, Les manifestations de rue en France, 1918-1968, Paris, Publications de la Sorbonne, 1997, $869 \mathrm{p}$.

TARTAKOWSKY Danièle, Les droites et la rue : histoire d'une ambivalence de 1880 à nos jours, Paris, La Découverte, 2014, 221 p. TCHAKHOTINE Serge, Le viol des foules par la propagande politique, Paris, Gallimard, 1952, 605 p. UGARTE Juana, « Deux grands hymnes idéologiques : le Te Deum, l'Internationale », Mots. Les langages du politique, vol. 70, 2002, en ligne.

VOVELLE Michel, « La Marseillaise, la guerre ou la paix », NORA Pierre (dir.), Les lieux de mémoire, T. 1 La République, Paris, Gallimard, 1997, pp. 107-149.

\section{NOTES}

1. CORBIN Alain, « Préface », CORBIN Alain, GÉRÔME Noëlle \& TARTAKOWSKY Danielle (dir.), Les usages politiques des fêtes aux $19^{e}-20^{e}$ siècles, Paris, Publications de la Sorbonne, 1994, p. 7. 
2. Ibid.

3. QUÉNIART Jean, Le chant, acteur de l'histoire, Rennes, Presses universitaires de Rennes, 1999.

4. BERSTEIN Serge, Le 6 février 1934, Paris, Gallimard/Juillard, 1975 ; TARTAKOWSKY Danielle, "Stratégies de la rue 1934-1936 », BOUVIER Jean (dir.), La France en mouvement 1934-1938, Seyssel, Champ-Vallon, 1986, pp. 31-60 ; TARTAKOWSKY Danielle, Les manifestations de rue en France, 1918-1968, Paris, Publications de la Sorbonne, 1997 ; TARTAKOWSKY Danièle, Les droites et la rue : histoire d'une ambivalence de 1880 à nos jours, Paris, La Découverte, 2014.

5. FERRO Marc, L'Internationale: histoire d'un chant de Pottier et Degeyter, Paris, Agnès Viénot, 1996.

6. BUCH Esteban, La Neuvième de Beethoven : une histoire politique, Paris, Gallimard, 1999 ; BUCH Esteban, «Les hymnes», DUCLERT Vincent \& PROCHASSON Christophe (dir.), Dictionnaire critique de la République, Paris, Flammarion, 2007, pp. 896-901.

7. PERROT Michelle, Les ouvriers en grève: France 1871-1890 (2 Vol.), Paris, Mouton \& Co, 1974.

8. VOVELLE Michel, «La Marseillaise, la guerre ou la paix », NORA Pierre (dir.), Les lieux de mémoire, T. 1 La République, Paris, Gallimard, 1997, pp. 107-149.

9. DARRIULAT Philippe, La Muse du peuple : chansons politiques et sociales en France 1815-1871, Rennes, Presses Universitaires de Rennes, 2010.

10. MORAT Daniel, "Cheer, Songs, and Marching Sounds: Acoustic Mobilization and Collective Affects at the Beginning of World War I ", MORAT Daniel (Dir.), Sounds of Modern History : Auditory cultures in $19^{\text {th }}$ and $20^{\text {th }}$ - century Europe, New-York/Oxford, Berghahn Books, 2017 [2014], pp. 177-200.

11. Une recherche doctorale est actuellement menée par l'auteur sur l'émergence du disque politique en France dans les années 1930.

12. L'usage est alors réputé ancien aux États-Unis et en Angleterre, mais il est tout récent en France. Ici, un haut-parleur est monté sur une voiture, et un orateur l'utilise pour s'adresser à des ouvriers à leur sortie d'usine. Par ailleurs, une firme cinématographique loue à la journée des haut-parleurs montés sur des tracteurs avec remorque, et le personnel adéquat, à des candidats aux élections qui se les arrachent. Voir MONTARON, « La voix qui parle aux foules... Un essai de réunion électorale avec un haut-parleur. », Le Populaire, 26 avril 1924, p. 1

13. TARTAKOWSKY, Les manifestations de rue en France, $869 \mathrm{p}$.

14. «Quel que soit le moyen employé, il s'agit [...] de manifester contre l'Allemagne et l'armée d'occupation en agissant de façon à s'assurer la sympathie de la foule. [...] Toute latitude est laissée aux éléments de la base à cet effet. De nombreux conseils leur sont donnés seulement à titre d'indication, par exemple : - se promener par petits groupes ou en chantant en sourdine le refrain de "La Marseillaise" et aussi celui de "L'Internationale". » Compte-rendu du 13/07/1941, B-37, BA 1917, APP. Par ailleurs, à la sortie d'une réunion du Progrès social français, "à la demande de M. Pradon, l'assistance a chanté "La Marseillaise" en sourdine et la sortie s'est effectuée sans incident. » Compte-rendu du 25/01/1941, 79.501-2726, BA 1952, APP.

15. Ces documents sont conservés dans les Archives de la Préfecture de Police de Paris (APP).

16. BERLIÈRE Jean-Marc, Le préfet Lépine: vers la naissance de la police moderne, Paris, Denoël, 1993, p. 54.

17. Ibid., p. 56.

18. Ibid., p. 162. 
19. TARTAKOWSKY, Les manifestations de rue, p. 398-414.

20. CERTEAU Michel DE, L'invention du quotidien. Tome 1 : arts de faire, Paris, Gallimard, 1990. Cette référence à ce point du travail de Michel de Certeau et son articulation au texte de Louis Marin, évoquée plus bas, sont empruntées à cet article: SIAUD Florent, "Liturgies de la rue», Tracés. Revue de Sciences humaines, vol. 5, 2004 (http:// traces.revues.org/3203, consulté le 30 septembre 2016).

21. CERTEAU, L'invention du quotidien, p. 172.

22. Ibid.

23. Comme, par exemple, la prise physique et symbolique d'un des lieux du pouvoir depuis l'espace de la rue, ou la démonstration de la cohésion d'un groupe militant massif, qui revendique ainsi sa légitimité à dominer la fabrication politique du sociétal.

24. Et ses abords, quand le son du meeting est diffusé à l'extérieur par des haut-parleurs.

25. « Rassemblement Populaire Antifasciste », 14/07/1935 à 16 h 15, BA 1861, APP.

26. « Manifestation du Front Populaire », 16/02/1936 à 17 h 40, BA 1862, APP.

27. « $\mathrm{N}^{\circ} 13$; Manifestation organisée par le Comité de Front Populaire ; cortège $\mathrm{n}^{\circ} 2$; $4^{\mathrm{e}}$ groupe », 14/07/1937 à 14 h 5, BA 1867, APP.

28. «P.M.A. ", 18/03/1937 à 11 h 5, BA 1865, APP.

29. Un exemple parmi d'autres: "Les participants crient "Thorez au pouvoir", "Fascistes assassins!" et chantent aussi l'Internationale et la Carmagnole.». " $\mathrm{N}^{\circ} 60$; Manifestation organisée par le Comité de Front Populaire ; cortège no 3 », 14/07/1934 à 15 h 45, BA 1867, APP.

30. "Manifestation des Travailleurs des Services Publics aux abords de l'Hôtel de Ville", 29/12/1936 à 18 h 10, BA 1864, APP.

31. BUCH, La Neuvième de Beethoven, p. 40.

32. Outre des chanteurs populaires, interprètes de chants politiques ou distractifs, il n'est pas rare que des chanteurs appartenant à l'Opéra, à l'Opéra-Comique ou à la Gaité Lyrique se produisent dans un répertoire lyrique lors de fêtes politiques rassemblant plusieurs milliers de personnes.

33. «Dans le $11^{\mathrm{e}}$ groupe, se trouvaient une centaine de militants communistes. [...] [Ils] chantaient, tant bien que mal, l'hymne italien révolutionnaire "Bandiera Rosa". " in « Rassemblement Populaire antifasciste », 14/07/1935 à 19 h 40, BA 1861, APP.

34. MORAT, « Cheers, Songs and Marching sounds », p. 178.

35. « P.M.A. ", 18/03/1937 à 11H05, BA 1865, APP.

36. « $\mathrm{N}^{\circ} 38$; Manifestation du Comité de Front populaire ; cortège $\mathrm{N}^{\circ} 2$ », 14/07/1937 à 14 h 55, BA 1867, APP.

37. « Sans titre », 23/06/1936, BA 1862, APP.

38. «Durant la concentration, sur le parcours du Cours de Vincennes à la Place de la Nation et dans l'intervalle des discours les participants ne manqueront pas, comme en tout circonstance semblable, de faire entendre des hymnes révolutionnaires et de pousser leurs cris habituels " in "Manifestation organisée place de la Nation par les Régions de Paris du Parti Communiste et les Fédérations de la Seine et de la Seine-etOise du Parti Socialiste (S.F.I.O.) ", 07/07/1934, BA 1860, APP. "Cette cérémonie coïncidera avec la sortie du personnel des bureaux et des magasins. Ainsi est-il possible que quelques centaines de personnes, attirées par les annonces des journaux d'extrême gauche, ou par simple curiosité, se rassemblent à l'entour. Il se peut que quelques-unes d'entre elles se laissent aller à pousser des cris d'hostilité à la guerre et au fascisme, et même à entonner des chants révolutionnaires. " in "Cérémonie commémorative de la mort de Jaurès le 31 Juillet, rue du Croissant », 28/07/1934, 264 500-115, BA 1860, APP. "L'organisation de ce banquet ne parait devoir susciter aucun incident. Il est 
seulement possible que l'issue en soit marquée par quelques cris ou chants royalistes. » in « Sans titre ", 16/03/1939, 340.700-28- ?, BA 1896, APP.

39. "Manifestation organisée par le Comité du Rassemblement populaire en réplique à l'agression commise contre Léon Blum. », 15/02/1936, BA 1862, APP.

40. CHION Michel, "The three listening modes ", STERNE Jonathan (dir.), Sound Studies Reader, Oxon, Routledge, 2012, pp. 48-53.

41. "Causal listening, the most common, consists of listening to a sound in order to gather information about its cause (or source)." CHION, « The three listening modes », p. 48.

42. « Rapport (Rage)», 19/02/1936, BA 1896, APP.

43. « Rapport (Torlet) », 19/02/1936, BA 1896, APP. Voir dans cette boîte les archives de la police parisienne relatives à la dissolution de la Ligue d'Action française et de ses associations.

44. La présence d'insignes et d'enseignes est toutefois plus particulièrement surveillée, et fait même l'objet d'instructions qu'envoie le Préfet de Police au Directeur Général de la Police Municipale et aux directeurs de la Police Judiciaire et des Renseignements généraux. Le chant n'y est pas mentionné. Voir « Instructions relatives à l'application du Décret de dissolution des ligues d'Action Française », 21/02/1936, BA 1896, APP

45. TARTAKOWSKY, Les droites et la rue, p. 63.

46. BERSTEIN, Le 6 février 1934, p. 164. Ce caractère apaisant pris alors par la Marseillaise est en quelque sorte revendiqué par Georges Lebecq, président de l'UNC, comme pour justifier face à la «Commission d'enquête sur les événements du 6 février » des chants entonnés par ses troupes : «À 20 heures, nous avons vu même circuler un camion portant un mort. Au même moment, nous donnons l'ordre de marche. Nous descendons les Champs Elysées en chantant la Madelon et la Marseillaise, et je vous assure qu'il était touchant d'entendre d'une seule voix ce chant patriotique. » in «Commission d'enquête sur les évènements du 6 février ; Séance du Mardi 27 Mars 1934 ; Présidence de M. Bonnevay ; La séance est ouverte à 14 h 45. », BA 1859, APP.

47. SCHAEFFER Pierre, Traité des objets musicaux : essai interdisciplines, Paris, Le Seuil, 1966, p. 122.

48. «Commission d'enquête sur les évènements du 6 février ; Rapport de la $3^{\mathrm{e}}$ sous-commission sur les conditions dans lesquelles le Service d'ordre a tiré à $19 \mathrm{~h} 30$ au pont de la Concorde », non daté, BA 1859, APP.

49. MARIN Louis, De la représentation, Paris, Le Seuil, 1994, p. 48.

50. тснакнотіNE Serge, Le viol des foules par la propagande politique, Paris, Gallimard, 1952, p. 272.

51. CANETTI Elias, Masse et puissance, Paris, Gallimard, 1966, p. 16.

52. CANETTI, Masse et puissance, p. 17.

53. SCHAFER Murray, Le paysage sonore : le monde comme musique, Paris, Wildproject, 2010, pp. 92 et 96.

54. CANETTI, Masse et puissance, p. 76.

55. Ibid.

56. «Un groupe de 200 personnes environ - dont une centaine chantaient la Marseillaise qui avaient été signalé boulevard St. Germain, se dirigeant vers le Bd. St. Michel, s'est dispersé sans incident. » in « Manifestation du Front Populaire », 16/02/1936 à 18 h 5, BA 1862, APP.

57. « Manifestation du Front Populaire », 16/02/1936 à 17 h 20, BA 1862, APP.

58. « Manifestation du Front Populaire », 16/02/1936 à 17 h 15, BA 1862, APP.

59. « $\mathrm{N}^{\circ} 70$; Manifestation organisée par le Comité de Front Populaire; Place de la Nation», 14/07/1937 à 17 h 15, BA 1867, APP. 
60. «Rapport ; Le Commissaire d'Arrondissement IV ${ }^{\mathrm{e}}$, à Monsieur le Directeur Général de la Police Municipale », 16/07/1935, 264.500-117-10, BA 1861, APP.

61. « Manifestation du Front Populaire », 16/02/1936 à 17 h, BA 1862, APP.

62. « P.M.A. ", 22/06/1936 à 19 h 10, BA 1862, APP.

63. « À la Gare Saint-Lazare. »12/07/1936, BA 1952, A.P.P.

64. «- 469 - », 04/10/1936, 264.500-122-2, BA 1863, A.P.P.

65. «Manifestation organisée par le Comité du Rassemblement Populaire en réplique à l'agression commise contre Léon Blum », 17/02/1936, BA 1952, A.P.P.

66. «Meeting commémoratif de l'anniversaire de la Commune de Paris, organisé par le Parti Communiste; Palais de la Mutualité, 24 rue St-Victor, le 20 Mars », 21/03/1936, 13.400-23, BA 2038, APP.

67. " 4 e Circonscription - Canton de st-Denis; Candidature Doriot; Salle du Théâtre municipal à Saint-Denis », 21/04/1932, 8 310, BA 1945, APP.

68. " 4 e Circonscription - Canton de st-Denis; Candidature Doriot; Salle du Théâtre municipal à Saint-Denis », 14/04/1932, 8 310, BA 1945, APP.

69. «Meeting organisé par la Société des Amis de l'U.R.S.S. pour commémorer le XVIII ${ }^{\mathrm{e}}$ anniversaire de la Révolution russe ; Salle Wagram - Le 7 Novembre », 08/11/1935, BA 1898, APP.

70. Voir notamment de nombreux comptes rendus de fêtes politiques partisanes ou associatives dans les boîtes d'archives de l'APP : BA 1896 ; BA 1898 ; BA 1939 ; BA 1956 ; BA 2038.

71. Voir ces deux comptes rendus: "Sans titre", 29/03/1936, 79.501-851, BA 1898, APP ; "Réunion organisée par les “Amis de l'U.R.S.S.”; Salle Wagram - le 29 Mai », 30/05/1936, 79.501-851, BA 1898, APP. Dans le premier, la police ne reconnaît « aucun caractère subversif » au programme musical d'une soirée exclusivement consacrée aux chants et musiques soviétiques. Dans le second, elle note le déroulement d'une partie artistique «n'ayant aucun caractère révolutionnaire » à la fin d'une réunion des Amis de l'Union soviétique. La police semble ainsi orienter son attention vers les menaces les plus directes de troubles, et négliger le rôle politique, ici d'adhésion culturelle, plus discret, profond et durable que peut jouer une musique sans caractère séditieux explicite.

72. "Fête de charité des dames royalistes. 8 rue Jean Goujon. Orateur: Real del Sarte. 300 personnes ", non daté, 340.700-28-B, BA 1896, APP. Cette archive, et celle qui la suit dans ce paragraphe, montre les limites informatives des rapports de police. Elle ne mentionne aucune date - la fête se déroule le 18 février 1939 - et «l'hymne franquiste » chanté est en fait un chant des phalanges - Volveron Banderas Gloriosas. Voir l'article du journal L'Action Française qui rapporte la fête : Non signé (1939), «Fête des Dames royalistes du XVII " $" 18$ février, p. 2.

73. «Melle Attard chante l'hymne franquiste». Le seul autre renseignement mis en valeur dans ce compte-rendu est « M. Real del Sarte fait l'éloge des camelots du roi disparus ». Ibid.

74. Ibid.

75. Il est encore rappelé, à la droite du corps du texte, le «Concours du chanteur nègre Paul Robson ». « Information; Réunion du Comité National allemand et de l'association des Ecrivains allemands 44 rue de Rennes; Ppx orateurs: Bode Uhse et Kurt Stern; Aide à l'Espagne Républicaine », 11/02/1938, BA 1970, APP.

76. «Réunion organisée par le Comité national allemand pour l'aide à l'Espagne et l'association des écrivains allemands - Salle de la Société d'encouragement de l'industrie Nationale, 44, rue de Rennes - Le 11 février - », 12/02/1938, BA 1970, A.P.P.

77. Voir le programme de la commémoration de la Commune par le PCF le 23/03/1939, où sont présentés les noms des compositeurs Grétry, Litolff, Gossec, Bizet, Schumann, Félicien David, Pierre Dupont, Liszt et Berlioz. «L'humanité 20/03/39 », 13.400-23, BA 2038, APP.

78. «Soirée artistique privée organisée par la section de Neuilly du Mouvement Social Français des Croix de Feu. », 14/02/1936, 79.501-1108-5, BA 1902, A.P.P. 
79. «Rapport; Le Commissaire d'Arrondissement IV e à Monsieur le Directeur Général de la Police Municipale », 16/07/1935, 264.500-117-10, BA 1861, APP.

80. Voir Rivière Claude (1988), Les liturgies politiques, Paris, Presses Universitaires de France, 256 p.

81. TARTAKOWSKY, Les manifestations de rue, p. 305. Ce qui ne signifie pas pour autant que la dimension religieuse des fêtes de droites ne procède que de la proximité de cet univers politique avec l'église, ni que son cas résume toute la similarité des pratiques et des opérations symboliques mises en œuvre par le religieux et le politique. Les usages de la voix - parlée, criée ou chantée - constituent d'ailleurs un de leurs principaux champs communs de pratiques. Pour son exploration, voir PoIZAT Michel, Vox Populi, Vox Dei : voix et pouvoir, Paris, Métaillié, 2001. Pour quelques exemples d'autres rituels politiques partageant leurs principes directeurs avec les rituels religieux, voir : MERCIER Arnaud, "Efficacité du performatif dans les rituels politiques », Hermès, La Revue, vol. 3, $\mathrm{n}^{\circ} 43,2005$, p. 31-37 (https://www.cairn.info/revue-hermes-larevue-2005-3-page-31.htm, consulté le 18 juin 2018).

82. Sans titre, 31/03/1940, BA 1952, A.P.P.

83. " $\mathrm{Au}$ sujet de la démonstration "Contre la Guerre Impérialiste" à Saint-Denis. », 31/07/1932, 62.909-78, BA 1945, A.P.P.

84. «Assemblée Populaire organisée par le Secours Rouge International. Salle des Fêtes - Mairie de Bagnolet, le 5 juillet », 05/07/1935, 20.965-B, BA 1861, A.P.P.

85. Voir à ce sujet Ferro 1996 et Vovelle 1997.

86. «L'émotion se manifeste plutôt à droite à l'occasion du rassemblement du 14 juillet. Dans les groupements du Front National, on se montre scandalisé à l'idée de voir le drapeau tricolore et le drapeau rouge côte à côte, salués par l'“Internationale" et la "Carmagnole". " in "Sans titre ", 01/07/1935, BA 1861, A.P.P.

87. BECKER Jean-Jacques \& BERSTEIN Serge, Histoire de l'anti-communisme en France, Paris, Orban, 1987.

88. ORY Pascal, «De "Ciné-Liberté" à La Marseillaise : espoirs et limites d'un cinéma libéré (1936-1938)», BOUVIER Jean (dir.), La France en mouvement 1934-1938, Seyssel, Champ-Vallon, 1986, p. 286.

89. « Réunion organisée par le Comité national allemand pour l'aide à l'Espagne et l'association des écrivains allemands - Salle de la Société d'encouragement de l'industrie Nationale, 44, rue de Rennes - Le 11 février - », 12/02/1938, BA 1970, A.P.P.

90. UGARTE Juana, «Deux grands hymnes idéologiques: le Te Deum, l'Internationale », Mots. Les langages du politique [En ligne], vol. 70, 2002 (http://mots.revues.org/8923, consulté le 11 octobre 2012).

91. FERRO, L'Internationale, p. 46.

92. Ibid., p. 64.

93. BUCH, La Neuvième de Beethoven, p. 33.

94. L'apprentissage de ses trois premiers couplets dans les écoles françaises est par ailleurs recommandé par voie ministérielle en 1911. Voir ALTEN Michèle, «Un siècle d'enseignement musical à l'école primaire ", Vingtième Siècle, revue d'histoire, nº 55, 1997, pp. 3-15.

95. BOURDIEU Pierre, Langage et pouvoir symbolique, Paris, Le Seuil, 2001, p. 63.

96. $8^{\mathrm{e}}$ Congrès du PCF. 1ere séance: Interventions de Gitton, Levy, Cachin, Bordes, Roqueblave, Chaumeil, Christofol, Bartholini. 2e séance: Interventions de Tillon, Monmousseau, Thorez. (22/01/1936), p. 266, Bobine 3 MI 6/ 119_751, Archives 
départementales de Seine-Saint-Denis. Document consultable en ligne à cette adresse : https://pandor.u-bourgogne.fr/img-viewer/PAPRIKA/000517/000001/001770/ viewer.html?ns=0517_0001_1770_0184.jpg [Consulté le 6 juin 2018].

97. MARIN, De la représentation, p. 55.

98. VOVELLE, « La Marseillaise, la guerre ou la paix », p. 146.

99. FERRO, L'Internationale, p. 51.

100. Voir infra.

\section{RÉSUMÉS}

À Paris, dans les années 1930, pourquoi les manifestants et les militants chantent-ils lorsqu'ils se rassemblent dans la rue, ou qu'ils se réunissent lors de meetings ou de fêtes politiques? Et pourquoi la police, qui surveille la vie politique partisane comme le lait sur le feu, ne cesse de faire mention du chant dans ses rapports et ses comptes rendus? À partir d'une analyse qualitative des archives policières de la surveillance politique des années 1930, cet article tentera de répondre à ces deux questions. Il considèrera déjà qu'il existe un imaginaire de la puissance politique du chant, qui motive ses usages par les militants, lui attribue des effets sociaux et attire sur lui l'attention de la police. Puis il tentera de renseigner cet imaginaire, à travers l'exploration d'un corpus d'usages politiques du chant. Pour enfin investir son fonctionnement politique, cet article proposera ensuite quelques routines de la manipulation du chant par ses chanteurs, celles-ci leur désignant leurs possibilités d'action sur une situation politique pratique. Il tentera ainsi de comprendre comment et à quelles fins le chant peut être employé pour servir de façon déterminante une pratique politique.

\section{INDEX}

Mots-clés : chant politique, manifestation politique, meeting politique, années 1930, imaginaire, surveillance politique, police, Internationale, Marseillaise

Keywords : political singing, political demonstration, political meeting, 1930s, imagination, political surveillance, Police, Internationale, Marseillaise.

\section{AUTEUR}

\section{JONATHAN THOMAS}

Jonathan Thomas est doctorant contractuel au Centre de recherche sur les arts et le langage (EHESS-CNRS). Il travaille, sous la direction du professeur Esteban Buch, sur le disque politique (France, 1929-1940). Il a publié des articles sur les usages politiques de la musique, du chant et du disque (SERP, Chant du Monde) dans les revues Volume ! et Analitica. 